Original Article (short paper)

\title{
Neuromuscular and physiological responses to different training loads in Randori of elite judo athletes
}

\author{
Bruno T. Campos ${ }^{1,2}$ (D), Eduardo M. Penna ${ }^{2,3}$ (D), Alexandre T. Katsuragi ${ }^{1}$, Leonardo Porto ${ }^{1}$, \\ Fúlvio K. Miyata ${ }^{1}$, Maicon Rodrigues Albuquerque ${ }^{1}$, Victor S. Coswig ${ }^{3}$ (D), Carolina F. Wilke ${ }^{1,2}$, \\ Luciano S. Prado ${ }^{2}$ (D) \\ ${ }^{1}$ Minas Tênis Clube, Departamento de Ciências do Esporte, Belo Horizonte, MG, Brasil. \\ ${ }^{2}$ Universidade Federal de Minas Gerais, Belo Horizonte, MG, Brasil. \\ ${ }^{3}$ Universidade Federal do Pará, Castanhal, PA, Brasil.
}

\begin{abstract}
Aim: To compare two different randori structure (high volume and short pauses - TRAD training vs low volume with long pauses - COMP training) in the neuromuscular and metabolic responses of elite judo athletes. Methods: The first situation (TRAD) consisted of 12 randoris of 5 minutes with 45 seconds rest, and the other situation (COMP), consisted of 6 randoris of 5 minutes with 10 minutes between them. Physiological (Blood Lactate and Creatine Kinase), neuromuscular (Countermovement Jump and Medicine Ball Throws) and perceptive variables (Rating Perceived Exertion) were measured before and at the end of the sessions. Results: The subjective perception of effort at the end of the training was significantly higher in the TRAD training type (TRADpost: $8.1 \pm 0.9 \mathrm{AU}$; COMPpost: 6.6 $\pm 1.5 \mathrm{AU}, \mathrm{p}<0.001$ ). Blood lactate concentration (TRADpost: $6.4 \pm 2.2 \mathrm{mmol} / \mathrm{L}$; COMPpost: $8.1 \pm 2.9 \mathrm{mmol} / \mathrm{L}$; $\mathrm{p}<0.001$ ), CMJ height (TRADpost: $36.2 \pm 4.6 \mathrm{~cm}$; COMPpost: $35.9 \pm 4.3 \mathrm{~cm}, \mathrm{p}=0.012$ ) and upper limb power performance (TRADpost: $6.4 \pm 0.9 \mathrm{~m}$; COMPpost: $6.3 \pm 0.9 \mathrm{~m} \mathrm{p}=0.03$ ) were significantly higher at the end of the two training sessions compared to their start, but there are no group effect. There is group effect in CK (TRADpost: $490.7 \pm$ 273.5 U $\backslash L$; COMPpost: $357.4 \pm 203.8 \mathrm{U} \backslash \mathrm{L} ; \mathrm{p}<0.001)$. Conclusions: This study demonstrated that TRAD and COMP Judo training induced similar physical demands, and both seem to not be enough to reach higher intensities, which made them distant methods of competitive reality.
\end{abstract}

Keywords: judo training, muscular power, muscle damage, fatigue.

\section{Introduction}

Judo is a combat sport that requires complex technicaltactical skills for success ${ }^{1}$. During each combat, athletes must perform several technical actions in a high-intensity intermittent time structure ${ }^{2}$, in which demands high metabolic and neuromuscular responses ${ }^{3,4}$. For instance, due to the grappling nature of the modality, judokas must grip the opponent's uniform (judogi), performing constant upper limbs isometric contractions; dominate their opponent, disrupt his/her balance and execute throwing techniques (nage-waza), in which requires powerful and short duration actions (1.0 to $1.4 \mathrm{~s}$ ) involving both lower- and upperbody muscle groups. When the combat takes place on the ground (ne-waza), technical actions require a combination of muscle power, anaerobic and strength endurance 5 .

Specific judo training is designed to prepare athletes to cope with such demands and improve performance ${ }^{6}$. Among the specific exercises, randori is the most used practice by coaches ${ }^{7}$. In addition, it was observed that $70 \%$ of the Olympic athletes performed the randori five to seven times a week in the final phase of preparation for the
Olympic Games ${ }^{8}$. Indeed, according to experienced coaches, the randori is an indispensable practice during the preparatory and competitive phase, being present in almost all sessions, usually during the last minutes of training sessions ${ }^{9}$.

Regarding the physiological demands of randoris, Branco et al. ${ }^{10}$ investigated the effect of 4 five-minute randoris with a five-minute pause in blood lactate concentrations, heart rate, and ratings of perceived effort (RPE). Results showed increases in blood lactate concentrations similar to official matches; increased heart rate in the $3^{\text {rd }}$ combat compared to the second and significant correlations between blood lactate concentration and RPE. Therefore, the randori can increase cardiovascular responses, induce muscle damage (creatine kinase; CK), and neuromuscular fatigue ${ }^{11,12}$. It is also known that neuromuscular fatigue after a match can be influenced by sev-

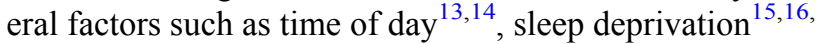
and type of recovery between matches ${ }^{17}$.

Importantly, these responses are thought to be modulated by the number and duration of this practice ${ }^{6}$. In practice routine, some coaches use randoris of high durations 
(longer than the official match time) with reduced recovery intervals (as the Japanese tradition). However, some coaches prefer a different approach, in which randori durations are similar to the competition durations (4-5 minutes). However, to the best of our knowledge, no study has investigated the effect of different randori structure (higher volume with short pauses with smaller volumes with long pauses) on acute neuromuscular and physiological responses. The manipulation of the randori duration and the recovery time between them could alter the metabolic pathway predominant in the session. Thus, by reducing the recovery time among the randori and increasing their number, we could increase the aerobic requirement and reduce the intensity of the match ${ }^{18}$. This knowledge should be of interest since training volume and rest intervals could be easily manipulated accordingly to different training goals and preparation phases.

Thus, this study aims to compare the effects of two different randori structures (high volume and short pauses -TRAD training vs low volume with long pauses - COMP training) on neuromuscular and physiological responses of elite judo athletes. It was hypothesized that COMP training would allow judokas to achieve higher intensities due to the longer recovery periods, which could induce higher RPE, blood lactate concentrations, more extensive muscle damage, and diminished post-exercise muscle power.

\section{Methods}

\section{Participants}

Sixteen male judo athletes (age: $22.0 \pm 3.0$ years; weight: $72.5 \pm 13.6 \mathrm{~kg}$; height: $170.0 \pm 6 \mathrm{~cm}$; body fat: 9 $\pm 2.2 \%$ ) volunteered to participate in this study. The judo experience was $7.2 \pm 3.9$ years. All athletes were black belts, had participated in several international and national tournaments, and were regularly training (technical and tactical training) 5 to 6 times a week during the evaluation period. They were in the preparatory phase and, therefore, were not in a period of rapid weight loss. Participants were instructed to avoid alcohol intake for at least 24 hours before experimental conditions and to maintain normal dietary habits. Before the assessments, all participants were informed about the procedures and signed an informed consent form. This study was approved by the Research Ethics Committee of the local university (Protocol number 12210219.7.0000.5149), following the Declaration of Helsinki. The participants were informed of the benefits and risks of the investigation before signing an institutionally approved informed consent document to participate in the study.

\section{Design}

Judo athletes participated in two types of judo training routines. The first condition was named traditional training (TRAD) and consisted of 12 randoris (combat simulation) of 5 minutes with 45 seconds rest intervals, simulating the most used practice routine in a daily practice of the studied squad. The second condition, named as competition training (COMP), consisted of 6 randoris of 5 minutes duration with 10 minutes rest intervals, simulating a typical judo competition. To assess the fatigue effects of each session, physiological, neuromuscular, and perceptive variables were measured before and at the end of the sessions.

\section{Procedures}

Before starting the training session, athletes were informed about the type of session (TRAD or COMP). The opponents of each match were predetermined considering similar body mass (difference of less than $10 \%$ among opponents) and competitive level. In case there was an ippon (punctuation that defines the fight), the combat was restarted to maintain the same duration for all the combats $^{19}$. All athletes participating in the study were already familiar with the test due to these being part of their testing routine of the training team they are part of. In addition, the athletes were stimulated during the randori to reach their maximum performance in all sessions.

At the start of the experimental procedures, the fingertip blood sample for lactate concentration was collected, and the, countermovement jump (CMJ) test and medicine ball throws were performed. In the two training sessions, a warm-up consisting of a 5-min free run with changes of direction and bearings and 20 repetitions of Uchikomis (repetition of a technique without projection) sets alternating with the partner was applied. After finishing the warm-up, the athletes rested for 5 minutes before starting the randori.

The experimental conditions were distributed in a randomized and balanced order. The tests batteries were always applied between $2 \mathrm{pm}$ and $4 \mathrm{pm}$, with a minimum interval of 48 hours between experimental situations, and the athletes were asked to not practice any physical exercise in the last 48 hours before the tests. The environmental conditions at the training center in which the tests were performed ranged between 18 to $22{ }^{\circ} \mathrm{C}$ temperature and 45 to $60 \%$ humidity.

\section{Countermovement jump}

The countermovement jump (CMJ) was performed on a flat and firm surface and with the assistance of a $0.1 \mathrm{~cm}$ precision contact mat (Hidrofit $\AA$ Ltda, Brazil) connected to Multisprint software (Hidrofit@ Ltda, Brazil). To perform the test, the athlete stood on the mat with his feet at a parallel position, hands resting on the height of the iliac crest, head up and looking forward. Athletes were instructed not to remove their hands from this position at any time during the jump, and that their knees should be extended during the flight phase. On hearing the "jump" 
command, the individual flexed their knee to an extent that felt more comfortable and jumped immediately upright as high as possible. During the tests, only jumps that met these requirements were considered for analysis. For the jump performance analysis, three attempts were performed and the highest value was used ${ }^{20}$.

\section{Medicine ball throws}

The muscle power of the upper limbs was assessed using the medicine ball throw performance. A 6-pound medicine ball was used. The athletes sat with their knees extended and their backs fully supported to the wall, holding the medicine ball close to the chest with their elbows flexed. At a signal, the athlete threw the ball as far as possible, keeping the back against the wall. The pitch distance was recorded considering the point where the ball touched the ground for the first time. Three throws were performed, and the best performance was registered for analysis.

\section{Blood lactate}

The collection of $20 \mu \mathrm{L}$ of blood from the digital pulp through heparinized capillary was performed at the beginning and the end of the randori organization and immediately inserted into the Accusport Lactate Analyser® portable lactimeter through reactive analysis tapes. The plasma lactate concentration in the sample being analyzed was estimated by its reflection value using an inbuilt algorithm. The instrument converted plasma readings to whole blood concentrations using an internal conversion factor. The Accusport analyzer was calibrated with a code strip that was specific for each package of 20 test strips for lactate determination.

\section{Creatine kinase (CK)}

Blood CK concentration was assessed by reflectance photometry at $37^{\circ} \mathrm{C}$ (Reflotron Plus; Roche, Germany), previously calibrated. After the finger asepsis by using alcohol, a 30- $\mu 1$ blood sample was drawn out into a heparinized capillary tube and it was later put on specific reagent strips that were inserted into the instrument.

\section{Ratings of perceived effort (RPE)}

The RPE at the end of the session was assessed using the Borg Category Ratio-10 (CR-10) RPE scale ${ }^{21}$ as an indication of the last randori ${ }^{\circ}$ s intensity. In addition, it is important to note that all athletes were trained in the use of the scale.

\section{Statistical analyses}

The normality and homoscedasticity of the data were confirmed prior to the inferential analysis by the ShapiroWilk and Levene test, respectively. For the inferential analysis, the significance level was set at $\alpha \leq 0.05$. Descriptive data are expressed as mean \pm standard deviation. A two-way ANOVA with repeated measures [two conditions (COM vs TRAD) and two moments (pre and post)] was performed for the physiological (CK and lactate) and neuromuscular (CMJ and Medicine Ball Throw) variables. When a significant $\mathrm{F}$ value was detected, the Bonferroni post-hoc test was used. For the non-normal distribution variable (RPE) the Wilcoxon test was used. Cohen's d effect size (ES) was calculated to assess the magnitude of the difference between the experimental trials. ES was calculated through mean differences and was considered as trivial $(\mathrm{ES}<0.2)$, small $(0.2<\mathrm{ES}<0.6)$, medium $(0.6<\mathrm{ES}<1.2)$, and large $(\mathrm{ES} \geq 1.2)^{22}$. All data were analyzed and plotted by the statistical package SigmaPlot11.0 (Systat Software Inc, USA).

\section{Results}

The subjective perception of effort at the end of the training was significantly higher in the TRAD training type $\left(\mathrm{TRAD}_{\text {post }}: 8.1 \pm 0.9 \mathrm{AU}\right.$; $\mathrm{COMP}_{\text {post }}: 6.6 \pm 1.5 \mathrm{AU}$, $p<0.001$; Figure 1).

Blood lactate concentration (Figure 2A) were significantly higher at the end of the two training sessions compared to their start $\left(\mathrm{TRAD}_{\text {post }}: 6.4 \pm 2.2 \mathrm{mmol} / \mathrm{L}\right.$; $\left.\mathrm{COMP}_{\text {post }}: 8.1 \pm 2.9 \mathrm{mmol} / \mathrm{L} ; \mathrm{F}: 84.549 ; p<0.001\right)$, with no group effect (F: $2.052, p=0.17, \mathrm{ES}=0.6$ ) or interaction (F: 3.196; $p=0.09)$ detected.

The two types of training significantly increased the creatine kinase concentration $\left(\mathrm{TRAD}_{\text {post }}: 490.7 \pm 273.5 \mathrm{U}\right.$ $\backslash \mathrm{L} ; \mathrm{COMP}_{\text {post }}: 357.4 \pm 203.8 \mathrm{U} \backslash \mathrm{L} ; \mathrm{F}: 36.148 ; p<0.001$ Figure $2 \mathrm{~B}$ ) and there was a group effect in which the TRAD training was significantly higher than the COMP training (F: $8.400 ; p=0.01$, ES=0.55), even though there was no significant interaction has been detected (F: 0.824, $p=0.37)$.

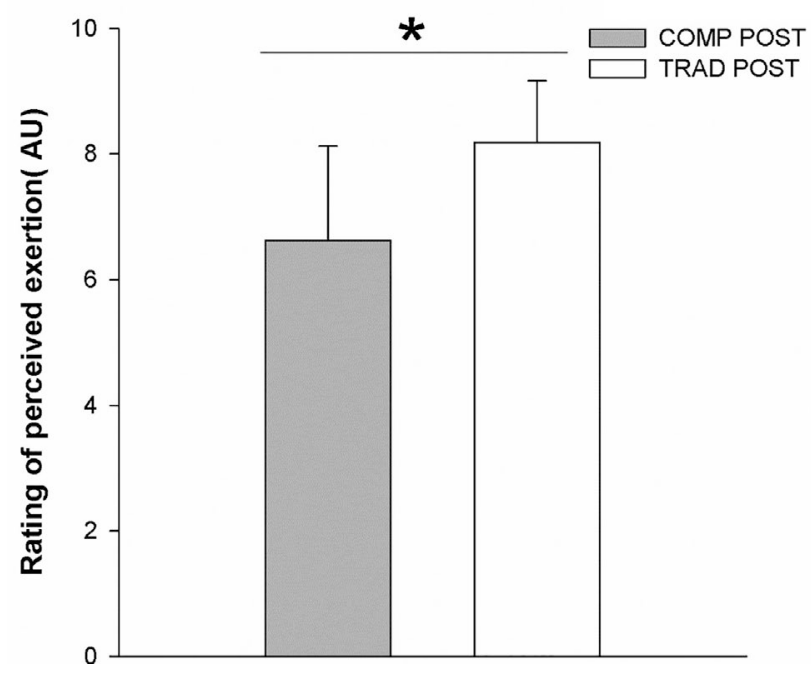

Figure 1 - Rating of perceived exertion. ${ }^{*} p<0.05$. 

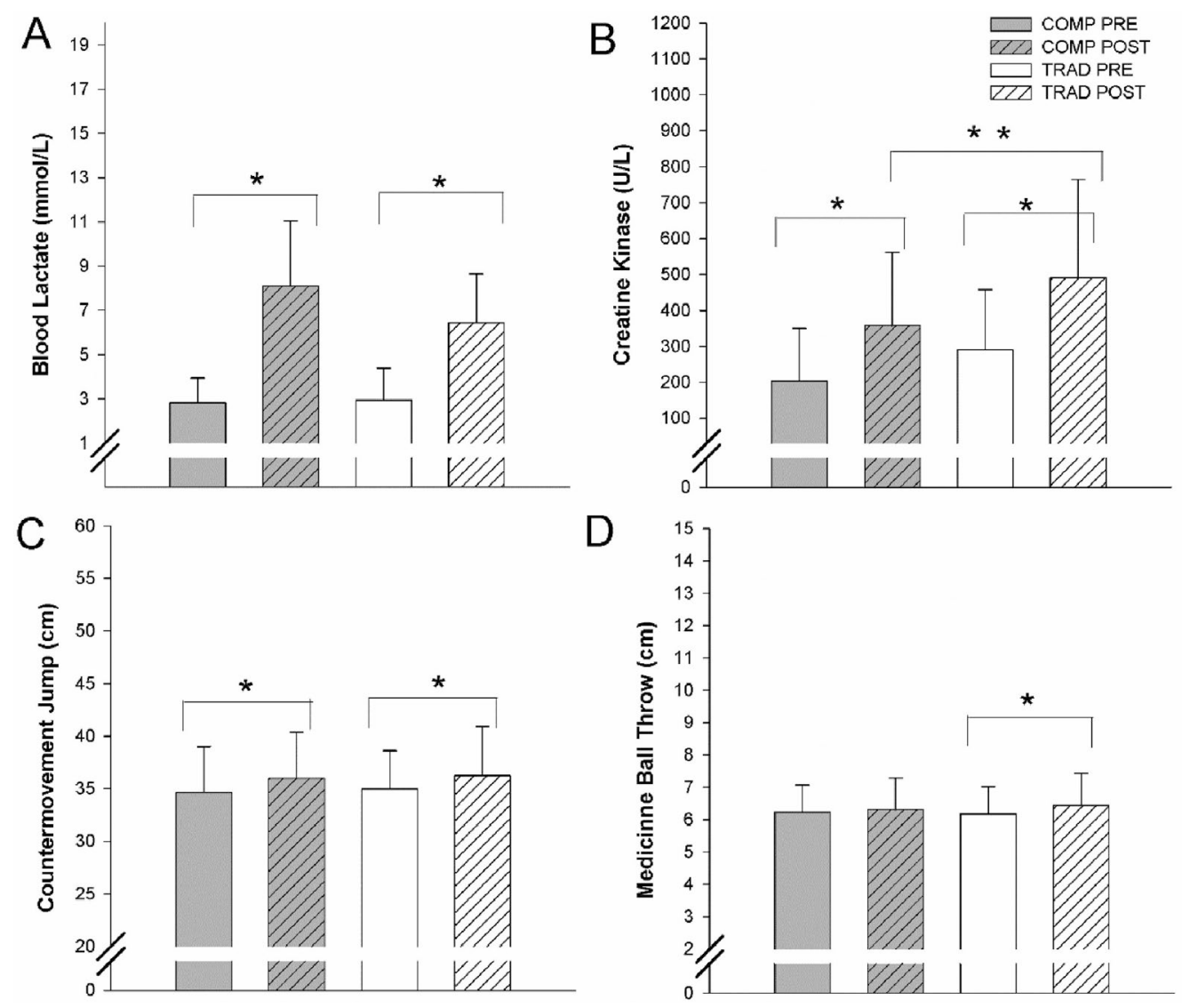

Figure 2 - Physiological and neuromuscular variables. *Time effect $(P<0.05) .{ }^{*}$ Group effect $(P<0.05)$.

CMJ height (Figure 2C) was higher at the end of the two training sessions (TRAD ${ }_{\text {post }}: 36.2 \pm 4.6 \mathrm{~cm}$; $\left.\mathrm{COMP}_{\text {post }}: 35.9 \pm 4.3 \mathrm{~cm}, \mathrm{~F}: 8.256 ; p=0.012\right)$, but there was no group effect (F: 0.302, $p=0.59$, $\mathrm{ES}=0.06)$ or significant interaction (F: 0.005, $p=0.94)$.

The analysis of upper limb power performance (Figure 2D) demonstrated higher values in the post-training compared to pre $(\mathrm{F}: 7.287 ; \mathrm{p}=0.01)$. There was no main group effect (F: $0.643 ; p=0.43$ ), though medicineball throw. Performance was higher at the end of the TRAD training compared to COMP (TRAD ${ }_{\text {post }}: 6.4 \pm$ $0.9 \mathrm{~m}$; $\mathrm{COMP}_{\text {post }}: 6.3 \pm 0.9 \mathrm{~m} p=0.03, \mathrm{ES}=0.1$ ).

\section{Discussion}

The aim of the present study was to compare two different randori structures (high volume and short rest intervals and low volume with long intervals) in the neuromuscular and physiological responses in elite judo athletes. We hypothesized that COMP condition would induce a higher subjective rating of perceived exertion, higher blood lactate concentrations, more pronounced muscle damage, and neuromuscular performance impairments than TRAD condition.
As not expected, RPE in COMP condition was lower than in TRAD condition. As previously documented, the RPE after judo combats increases linearly with the lactate concentration ${ }^{5}$. However, it seems that the TRAD training, due to the short recovery intervals, may have led to a higher requirement of the oxidative energy system, probably induced by the decreased ATP-CP contribution after the third minute ${ }^{18}$, which leads to a higher cardiovascular strain and consequently impacting how the athlete perceives the effort in this practice. Julio et $\mathrm{al}^{18}$ demonstrated that oxidative system to supply the energy cost of judo matches from the first minute of combat up to the end when compared to the anaerobic systems. With the development of combat, there was an increase in the aerobic contribution (from $50 \%$ to $81 \%$ ) with a concomitant reduction in the contribution of the ATP-PCr system (from $40 \%$ to $12 \%$ ), maintaining a low glycolytic system contribution (between $6 \%$ and $10 \%$ ). Finally, in the first minute of combat, there was a higher rate of the number of technical sequences with reduced pause time and higher values of total metabolic power per minute of combat.

Although there were no statistically significant differences between conditions in the lactate response, the effect size was medium for a higher lactate response after COMP training, indicating a possible higher intensity in 
COMP training than TRAD training in the last randori. Nevertheless, the mean lactate concentration in COMP training was $8.1 \pm 2.9$ mmol.L-1. Already been reported that after a specific judo training (uchikomis, ne-waza, and 3-7 randori) the lactate concentration reaches $9.1 \pm$ $1.1 \mathrm{mmol} . \mathrm{L}^{-123}$, while the average lactate in a judo competition is approximately $12 \pm 3.6 \mathrm{mmol} . \mathrm{L}^{-15}$. Therefore, the intensity performed in the training structure of the present study could be lower than the intensity of competition, and that new forms of organization of randoris should be conceived of in order to provide the achievement of higher intensities. However, this information should be considered with caution, since pre and post analysis solely limits the understanding of the production/removal lactate dynamics.

The present study founded that creatine kinase was significantly higher in COMP condition than in TRAD condition. Previous studies have shown that a judo session can lead to increased markers of muscle damage ${ }^{11,24}$, probably due to the eccentric-concentric actions that induce cytoskeletal and sarcolemma disruption ${ }^{12}$. A plausible explanation can be related to the total training time, in which the TRAD condition had twice the duration of the randori effort concerning COMP condition.

It was expected that the COMP condition would lead to a decrease in lower limb power production when compared to the TRAD structure. However, neither of the two training formats led to a reduction in lower limb power after training and no difference between training. Although some authors have already found lower limb power after combat simulations ${ }^{12}$, another study has not reported changes in the CMJ height after combats ${ }^{25}$. In some actions of judo, the athlete must perform a hip and knee flexion in the techniques and projection stages. This move is necessary for Uke to place his center of mass in a position under the center of mass of his opponent since it is the extension of the knees that will suspend the opponent ${ }^{26}$. However, in the present study, these actions may not have been carried out strongly by athletes in both training structures, not leading to a manifestation of fatigue of this capacity.

The present study also found no reduction in upper limbs power after COMP training. Contrary to our expectation, an increase in performance was observed after TRAD training. This result can be explained by the specificity of the force manifestation during the combat, in which, strength endurance is highly required due to grappling disputes ${ }^{5}$, consequently not affecting the ability of the neuromuscular system to perform power movements.

Another variable that may have influenced our results could be the effect of the time of day (chronobiology) on muscle power performance. Some authors have already shown better muscle power performance in the afternoon $^{15,27}$. The central temperature, strength, and muscle power of the judokas are dependent on the time of day, with higher values at 16:00 h. However, the daily variations of short-term anaerobic performances disappeared after the judo fight, indicating greater muscle fatigue at $16: 00^{13,14}$. Although as our study was conducted in the afternoon, this time of day effect maybe has not influenced the muscle power after training.

Some limitations should be considered while interpreting our findings. First, total effort time was not the same between training situations. Despite this may reduce internal validity, this was a methodological choice to guarantee external validity by the real and common Judo training practices. In addition, athletes may have controlled the randori pace in the TRAD method due to the longer duration. However, the athletes were stimulated during the randori to reach their maximum performance in all sessions. Another limitation of the study was the use of the match time of $5 \mathrm{~min}$. However, with the current change in the rule, in which the match has 4 min, new studies need to be designed based on this new time of match.

\section{Conclusions}

This study demonstrated that TRAD and COMP Judo training induced similar neuromuscular demands and different physiological responses. In addition, both conditions seem to not be enough to reach higher intensities, which made them distant methods of competitive reality. In this sense, based on our findings, the training method could be chosen based on specificity and periodization phases. For example, the TRAD method induced higher RPE, which means that it is not the best choice for tapering periods. On other hand, the COMP training did not produce physiological (i.e. blood lactate concentrations) or neuromuscular responses when compared to what is expected in the competition, which means that it could be considered as a technical-tactical/recovery session, but not as a conditioning session. In this sense, neither COMP or TRAD methods seem to properly simulate what is expected for competition. Thus, we suggest that coaches, trainers, and sports scientists should consider our findings while looking for what variables could be better manipulated to raise/lower randori intensity, which would allow new methods that could closely mimic the reality of the fight.

\section{References}

1. Miarka B, Branco BHM, Vecchio FBD, Camey S, Franchini E. Development and validation of a time-motion judo combat model based on the Markovian Processes. Int J Perform Anal Sport. 2015;15:315-31.

2. Sterkowicz-Przybycien K, Miarka B, Fukuda DH. Sex and Weight Category Differences in Time-Motion Analysis of Elite Judo Athletes. J Strength Cond Res. 2017;31(3):81725. 
3. Franchini E, Del Vecchio FB, Matsushigue KA, Artioli GG. Physiological profiles of elite judo athletes. Sports Med. 2011;41(2):147-66.

4. Fullagar HHK, Duffield R, Skorski S, White D, Bloomfield J, Kölling S, et al. Sleep, Travel, and Recovery Responses of National Footballers During and After Long-Haul International Air Travel. Int J Sports Physiol Perform [Internet]. 2016 Jan;11(1):86-95. Available from: https://journals.humanki netics.com/view/journals/ijspp/11/1/article-p86.xml

5. Franchini E, Artioli GG, Brito CJ. Judo combat: timemotion analysis and physiology. Int J Perform Anal Sport. 2013;13(3):624-41.

6. Franchini E, Brito CJ, Fukuda DH, Artioli GG. The Physiology of Judo-Specific Training Modalities. J Strength Cond Res [Internet]. 2013;0(0):1-8. Available from: http:// www.ncbi.nlm.nih.gov/pubmed/24149757

7. Pedrosa GF, Soares YM, Gonçalves R, Couto BP, Da Silva RAD, Szmuchrowski LA. Elaboration and evaluation of judo training means. Arch Budo. 2015;11:7-16.

8. Franchini E, Takito MY. Olympic Preparation in Brazilian Judo Athletes: Description and perceived relevance of training practices. J Strength Cond Res [Internet]. 2014;28 (6):1606-12. Available from: http://content.wkhealth.com/ linkback/openurl?sid=WKPTLP:landingpa ge\&an=00124278-201406000-00015

9. Pedrosa GF, Teobaldo B, Januário M, Soares YM, Chaves Filho AF, Alves GR, et al. Recording process of judo training. In: Proceedings of the 1st World Congress on Health and Martial Arts in Interdisciplinary Approach - Archives of Budo. 2015. p. 112-8.

10. Branco BHM, Massuça LM, Andreato L V., Marinho BF, Miarka B, Monteiro L, et al. Association between the rating perceived exertion, heart rate, and blood lactate in successive judo fights (Randori). Asian J Sports Med. 2013;4(2):125-30.

11. Umeda T, Yamai K, Takahashi I, Kojima A, Yamamoto Y, Tanabe M, et al. The effects of a two-hour judo training session on the neutrophil immune functions in university judoists. Luminescence. 2008;23(1):49-53.

12. Detanico D, Dal Pupo J, Franchini E, Dos Santos SG. Effects of successive judo matches on fatigue and muscle damage markers. J Strength Cond Res. 2015;29(4):1010-6.

13. Chtourou H, Aloui A, Hammouda O, Chaouachi A, Chamari K, Souissi N. The effect of time-of-day and judo match on short-term maximal performances in judokas. Biol Rhythm Res. 2013;44(5):797-806.

14. Chtourou H, Engel FA, Fakhfakh H, Fakhfakh H, Hammouda O, Ammar A, et al. Diurnal Variation of Short-Term Repetitive Maximal Performance and Psychological Variables in Elite Judo Athletes. Front Physiol [Internet]. 2018 Oct 26;9(OCT):1-7. Available from: https://www.frontier sin.org/article/10.3389/fphys.2018.01499/full

15. Souissi N, Chtourou H, Aloui A, Hammouda O, Dogui M, Chaouachi A, et al. Effects of time-of-day and partial sleep deprivation on short-term maximal performances of judo competitors. J Strength Cond Res [Internet]. 2013;27 (9):2473-80. Available from: http://www.ncbi.nlm.nih.gov/ pubmed/23974210

16. Hajsalem M, Chtourou H, Aloui A, Hammouda O, Souissi N. Effects of partial sleep deprivation at the end of the night on anaerobic performances in judokas. Biol Rhythm Res. 2013;44(5):815-21.
17. Hamdi C, Hana B, Asma A, Najla I, Liwa M, Karim C, et al. EFFECTS OF RECOVERY TYPE ON JUDOKAS' SHORT-TERM MAXIMAL PERFORMANCES DURING A SIMULATED COMPETITION. Br J Sports Med [Internet]. 2013 Jul;47(10):e3.8-e3. Available from: http://bjsm. bmj.com/lookup/doi/10.1136/bjsports-2013-092558.16

18. Julio UF, Panissa VLG, Esteves J V., Cury RL, Agostinho MF, Franchini E. Energy-System Contributions to Simulated Judo Matches. Int J Sports Physiol Perform [Internet]. 2017 May;12(5):676-83. Available from: https://journals.humanki netics.com/view/journals/ijspp/12/5/article-p676.xml

19. Franchini E, Dunn E, Takito MY. Reliability and Usefulness of Time-Motion and Physiological Responses in Simulated Judo Matches. J Strength Cond Res [Internet]. 2018 Jul;0 (0):1. Available from: http://insights.ovid.com/crossref? an=00124278-900000000-95243

20. Klavora P. Vertical-jump Tests?: A Critical Review. Strength Cond J. 2000;22(5):70-5.

21. Foster C, Florhaug JA, Franklin J, Gottschall L, Hrovatin LA, Parker S, et al. A new approach to monitoring exercise training. J Strength Cond Res. 2001;15(1):109-15.

22. Hopkins WG, Marshall SW, Batterham AM, Hanin J. Progressive statistics for studies in sports medicine and exercise science. Med Sci Sports Exerc. 2009;41(1):3-12.

23. Callister R, Callister RJ, Staron RS, Fleck SJ, Tesch P, Dudley GA. Physiological characteristics of elite judo athletes. Int J Sports Med. 1991;12(2):196-203.

24. Chinda D, Umeda T, Shimoyama T, Kojima A, Tanabe M, Nakaji S, et al. The acute response of neutrophil function to a bout of judo training. Luminescence. 2003;18(5):278-82.

25. Bonitch-Domínguez J, Bonitch-Góngora J, Padial P, Feriche B. Changes in peak leg power induced by successive judo bouts and their relationship to lactate production. J Sports Sci. 2010;28(14):1527-34.

26. Campos BT, Cabido CET, Soares YM, Pedrosa GF, Mendes TT, Almeida RSV de. A prior isometric strength exercise increases muscle power performance of judo athletes. J Phys Educ. 2018;29(1):1-9.

27. Chtourou H, Engel FA, Fakhfakh H, Fakhfakh H, Hammouda O, Ammar A, et al. Diurnal variation of short-term repetitive maximal performance and psychological variables in elite judo athletes. Front Physiol. 2018;9(OCT):1-7.

Corresponding Author: Bruno Teobaldo Campos Rua União 80, Jardim Alvorada, CEP: 33938-120, Ribeirão das Neves, MG, Brasil.

E-mail: brunoteobaldocampos@hotmail.com.br.

Manuscript received on November 7, 2019

Manuscript accepted on April 7, 2020

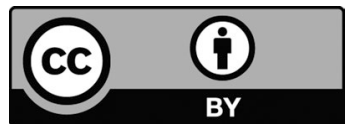

Motriz. The Journal of Physical Education. UNESP. Rio Claro, SP, Brazil - eISSN: 1980-6574 - under a license Creative Commons - Version 4.0 